\title{
I. Einleitung*
}

Als eine "Orgie der Gewalt, die in der Weltgeschichte ihresgleichen sucht", hat die sino-amerikanische Publizistin Iris Chang den grausamen Höhepunkt der japanischen Kriegsverbrechen in der alten chinesischen Hauptstadt Nanking 1937 bezeichnet und kritisiert, daß die meisten Nicht-Asiaten „über die Schrecken des Massakers anders als über die Atombombenexplosionen in Japan oder den Holocaust in Europa fast nichts" wüßten1. Tatsächlich wurde Nanking in wichtigen Standardwerken zur Geschichte des Zweiten Weltkriegs oder z.B. in den Churchill-Erinnerungen nicht einmal erwähnt. Und in Japan selbst wird Kriegsverbrechern im Tokioter Yasukuni-Schrein in einer Art und Weise gedacht, die ein überlebendes amerikanisches Opfer zu der Einschätzung veranlaßte, dies sei etwa so, als planten die Deutschen „mitten in Berlin eine Kathedrale für Hitler zu erbauen“2. Der ehemalige Ministerpräsident Singapurs Lee Kwan-Yew kam (1992) zu dem Schluß: „Unglücklicherweise sind die Japaner, anders als die Deutschen, im Umgang mit den von ihnen während des Zweiten Weltkriegs verursachten Greueltaten und furchtbaren Katastrophen weder offen noch ehrlich ...".3

Der Franzose Alfred Grosser urteilt ähnlich über die „Vergangenheitsbewältigung" in der Bundesrepublik Deutschland und den Genozid im Gedächtnis der Menschheit: „Wohl nirgend wo sonst auf der Welt hat eine Gemeinschaft in vergleichbarem Ausmaß akzeptiert und gewünscht, daß die dunkle Vergangenheit in der Gegenwart eine so zentrale Stellung einnimmt ${ }^{\text {"4 }}$. Die These des renommierten Friedenspreisträgers des deutschen Buchhandels hat indes wenig Resonanz gefunden. Dieser Eindruck drängt

Der Konvention entsprechend wird bei der Nennung japanischer Eigennamen im folgenden der Familienname vorangestellt. Lang gesprochene Vokale werden mit einem Hilfszeichen $(\wedge)$ über dem Buchstaben gekennzeichnet.

1 Chang, Die Vergewaltigung von Nanking, S. 11f. Die Fehler des Buches erleichterten es der japanischen Rechten, gegen die Publikation Front zu machen. Chang hatte unter anderem nicht richtig wahrgenommen, daß in Japan in den 1970er Jahren doch eine intensivere Debatte über Nanking begonnen hatte. Vgl. hierzu vor allem die Einleitung von Gibney in: Honda, The Nanjing Massacre, sowie Ishida, Das Massaker von Nanking, S. 235, und Bork, Geschichtsklitterung in Sprechblasen, in: Die Zeit, Nr. 11, v. 11. März 1999. Jüngere Publikationen zum Thema Nanking analysiert Yang, Re-Examining the Japanese Atrocities in Nanjing.

2 Chang, Die Vergewaltigung von Nanking, S. 18.

3 Zit. nach Tanaka, Japans Nachkriegsverantwortung, S. 411.

4 Grosser, Ermordung der Menschheit, S. 147. Ähnlich auch die Einschätzung von Schwarz, Epochenwechsel, S. 208. 
sich jedenfalls auf, wenn man etwa die einschlägigen Bibliotheksbestände des Instituts für Zeitgeschichte allein für den jüngsten Zeitraum ab 1995 sichtet. Unter dem Stichwort „Vergangenheitsbewältigung“ stößt man dabei auf mehrere Hundert, überwiegend deutschsprachige Bücher historischer, philosophischer, politologischer, soziologischer, psychologischer oder (volks-)pädagogischer Thematik, die aber selten einmal die deutsche Entwicklung mit der in anderen Ländern vergleichen, sondern sich allen möglichen Facetten der nationalen Erinnerung widmen. Diese Beobachtung ist allerdings nicht dahingehend mißzuverstehen, daß es nicht auch noch mehr als genug Forschungsdesiderata in bezug auf die nationale deutsche Auseinandersetzung mit der NS-Vergangenheit gäbe, man denke nur an das eminent wichtige, bis heute wissenschaftlich nicht umfassend geklärte Thema der strafrechtlichen Verfolgung von NS-Gewaltverbrechen durch die deutsche Justiz in den 1950er Jahren ${ }^{5}$. Richtig ist aber doch, daß es allzu lange, wenn überhaupt, Kolloquien und Sammelbänden überlassen worden ist $^{6}$, die Erinnerungsgeschichte an Nationalsozialismus und Zweiten Weltkrieg in der Bundesrepublik in eine international vergleichende Perspektive zu rücken?.

Trotz Hermann Heimpels gut begründeten Plädoyers aus den 1950er Jahren, „daß die Zeit einer ausschließlich nationalstaatlichen Geschichtsbetrachtung vorbei“ ${ }^{\circ}$ sei, hat sich an der primär nationalgeschichtlichen Orientierung, manche sagen gar: „hoffnungslosen Provinzialität“ deutscher Historiographie ${ }^{9}$ bis in die jüngste Zeit hinein nicht schrecklich viel geän$\operatorname{dert}^{10}$. Die Möglichkeit, das heuristische Potential international vergleichender Geschichtswissenschaft zu nutzen, stieß sich gerade beim Thema der NS-Vergangenheit und ihrer Bewältigung in Deutschland lange hart im Raume mit der Forderung, „nicht aufzurechnen“, die Schatten der eigenen

5 Dieser Schluß läßt sich etwa aus einem Projekt des Instituts für Zeitgeschichte zur Inventarisierung von Verfahrensakten ziehen. Vgl. hierzu Eichmüller, Die Verfolgung von NS-Verbrechen.

6 Vgl. etwa den Tagungsband von Knigge/Frei (Hg.), Verbrechen erinnern, mit einer leider nur ganz knappen Skizze der japanischen „Vergangenheitsbewältigung" von Sato, Japan und der Zweite Weltkrieg, sowie Bock/Wolfrum, Umkämpfte Vergangenheit, oder Weber/Piazolo, Justiz im Zwielicht.

7 Wie aufschlußreich ein Vergleich gerade auch höchst unterschiedlicher Erinnerungskulturen sein kann, hat am Beispiel Israels und Deutschlands Zuckermann, Zweierlei Holocaust, gezeigt.

8 Heimpel, Über Geschichte und Geschichtswissenschaft, S. 22.

9 Vgl. Eckert, Gefangen in der Alten Welt. Andreas Eckerts scharfe Kritik an dem Desinteresse deutscher Geschichtswissenschaft für außereuropäische Geschichte ist allerdings insofern ergänzungsbedürftig, als sie von dem ebenfalls noch nicht hinreichend ausgeprägten Forschungsinteresse an außerdeutschen europäischen Gegenständen absieht, das angesichts der Entwicklung zur Europäischen Union zumindest ebenso anachronistisch wirkt.

10 Die Situation der deutschen Geschichtswissenschaft sei vielmchr, so sehen es Heinz-Gerhard Haupt und Jürgen Kocka, durch höchste Spezialisierung und Kleinschrittigkeit gekennzeichnet. Haupt/Kocka, Historischer Vergleich, S. 26. 
Geschichte nicht durch den Blick auf die der Nachbarn perspektivisch zu verkürzen und dadurch womöglich den Prozeß der demokratischen Läuterung der bundesrepublikanischen Gesellschaft zu gefährden. So richtig es war, zunächst vor der eigenen Tür zu kehren, so deutlich ist doch heute auch, daß sich die deutsche historische Forschung zur „Vergangenheitsbewältigung“ im eigenen Land wichtiger Erkenntnismöglichkeiten begäbe, wenn sie in den „Grenzen eines nationalgeschichtlichen Reduktionismus"11 verharrte und nicht - von dem zwischenzeitlich erreichten Fundament eines fest in die westliche Wertegemeinschaft eingebundenen Staatswesens aus damit begänne, die unleugbaren Defizite ebenso wie die „Erfolge“ der eigenen „Vergangenheitsbewältigung“ im vergleichenden Blick auf ähnliche Prozesse in anderen Nationen differenzierter beurteilen zu lernen. Tatsächlich lassen sich Verdrängungssymptome und Erinnerungsleistungen einer Gesellschaft - auch der bundesdeutschen - „erst im Vergleich zu anderen präziser bestimmen" 12 .

Eine komparative Untersuchung des Genozids im Gedächtnis der Völker hat - wie beispielsweise Alfred Grosser deutlich macht - keineswegs von der Besonderheit des in Auschwitz verübten industrialisierten Massenmordes selbst abzusehen ${ }^{13}$. Gerade die Betonung der Singularität eines historischen Geschehens setzt ja paradoxerweise den Vergleich stillschweigend voraus, liegt doch „die Bedeutung einer Einzigartigkeit ... darin, daß alle anderen Phänomene im Vergleich $z u$ ibr durchschnittlich und in ihrer Durchschnittlichkeit untereinander vergleichbar sind“14. Man kann sich zudem fragen, ob das Beharren auf dem Unvergleichlichen nicht den Blick auf die Möglichkeit verstellte, „den systematischen Massenmord als einen Grundzug der modernen Zivilisation zu begreifen und somit die Perspektive, die Adorno und Horkheimer mit ihrer Dialektik der Aufklärung entwarfen, auf weitere Phänomene auch außerhalb der europäischen Geschichte anzuwenden" 15 ?

Gewiß ließe sich kategorisch argumentieren, aus der Sicht der Opfer sei jeder Vergleich von Verbrechen verwerflich, „weil die einzigartige Authentizität des historischen Geschehens dadurch bedroht wird." 16 . Andererseits gehen gerade komparatistische Ansätze insofern von einer Opferperspektive aus, als sie prinzipiell die Ermordung jedes einzelnen Menschen für gleich bedeutend und erinnerungswürdig halten, ob die Täter nun im Dienste einer vulgärdarwinistischen (nationalsozialistischen) Weltanschauung,

11 Darauf haben jetzt zu Recht Christoph Cornelißen, Lutz Klinkhammer und Wolfgang Schwentker hingewiesen. Cornelißen/Klinkhammer/Schwentker, Nationale Erinnerungskulturen, S. 20.

12 Ebd.

13 Grosser, Ermordung der Menscheit, v.a. S. 45 f., S. 95 f.

14 Shimada, Formen der Erinnerungsarbeit, S. 30.

15 Ebd., S. 31.

16 Ebd., S. 30. 
im Namen einer pseudohumanistischen (kommunistischen) Ideologie oder auf dem Boden eines „asiatischen“ Rassismus agierten und ob dabei neben diesem einzelnen Opfer nun einige Hundert oder viele Millionen Menschen ums Leben kamen. Oder, um es mit Albert Camus zu sagen: Jeder gewaltsame Tod ist ein Weltuntergang.

Im folgenden geht es aber ohnehin nicht um einen Vergleich von Genoziden und den sie verübenden Regimen, sondern um die Analyse unterschiedlicher Erinnerungskulturen. Zu den Bedingungen für deren Möglichkeit gehört keineswegs die absolute Gleichartigkeit der zu „bewältigenden“ Verbrechenskomplexe. Vielmehr reicht es hin, daß sich in der ersten Hälfte des 20. Jahrhunderts in mehreren großen Ländern, im stalinistischen Rußland wie im ultranationalistisch-militaristischen Japan, Herrschaftssysteme etabliert hatten, die ähnlich wie das nationalsozialistische Deutschland eine bis dahin nahezu unvorstellbare kriminelle Energie nach innen und/oder nach außen entwickelten und vor allem im Zeitalter des Zweiten Weltkrieges eine apokalyptische Zahl an (Todes-)Opfern produzierten.

Doch während der Vergleich der „Vergangenheitsbewältigung“ in der Bundesrepublik Deutschland mit der Japans auch deswegen naheliegt, weil sich hier nach dem Zweiten Weltkrieg ebenfalls ein Systemwechsel vollzog und eine demokratische Gesellschaft etablierte ${ }^{17}$, unterläge die Einbeziehung Rußlands der offensichtlichen Schwierigkeit, daß dies dort erst zögernd seit Gorbatschow bzw. seit dem Ende der Sowjetunion 1991 geschah, während andererseits schon Mitte der 1950er Jahre nach dem XX. Parteitag der KPdSU ein, wenn auch nicht demokratisch normierter Prozeß der Bewältigung des Stalinismus eingesetzt hatte. Zwar sind seit dem Zerfall der UdSSR mittlerweile auch schon über zehn Jahre vergangen - rein chronologisch gesehen also das Stadium der Bundesrepublik Mitte der 1950er Jahre erreicht -, was ein Urteil zumindest über die Take-Off-Phase der Bewältigung erlaubte. Zudem gehört es zum üblichen Instrumentarium komparatistischer Historiographie, auch zeitlich verschobene Phasen unter einem bestimmten tertium comparationis zu untersuchen ${ }^{18}$. Doch konzentriert sich der vorliegende Vergleich schon deshalb auf Japan, weil der internationale Forschungsstand zur Stalinismus-Bewältigung im neuen Rußland noch dürftiger ist als die Bewältigung selbst ${ }^{19}$.

17 Wenn auch von politologischer Seite darauf verwiesen wird, daß sich die oligarchischen Herrschaftsstrukturen Japans selbst durch die Zäsur von 1945 nicht entscheidend gewandelt hätten; allerdings hätten Demokratie, Entmilitarisierung und vermehrte Kontakte mit dem Westen zumindest die Bedingungen und Ziele dieser Herrschaft verändert. J. Hartmann, Politik und Gesellschaft, S. 55.

$18 \mathrm{Zu}$ den methodischen Herausforderungen international vergleichender Geschichtswissenschaft vgl. am Beispiel des vom Institut für Zeitgeschichte durchgeführten deutsch-französischen Projekts Kittel/Neri/Raithel/Wirsching, Faktoren der Stabilität und Instabilität.

19 Einen ersten Einblick in die Problematik vermitteln Fein, Geschichtspolitik in Rußland; Sunder-Plaßmann, Rettung oder Massenmord?; Ferreti, La mémoire refoulée. 
Im Mittelpunkt der Studie steht der Versuch, wesentliche Komplexe vor allem der frühen japanischen Auseinandersetzung mit der ultranationalistisch-militaristischen bzw. „Tennô-System-faschistischen“20 Vergangenheit anhand der internationalen und neuerdings endlich auch deutschen Forschung zu dem Thema einmal zumindest in großen Umrissen monographisch zusammenhängend darzustellen und die sich daraus ergebenden Befunde knapp mit den im wesentlichen als bekannt vorauszusetzenden (bundes-)deutschen Phänomenen zu vergleichen. Denn ein „systematischer und abgewogener Vergleich der Bewältigungsformen von deutscher Seite" steht, wie Volker Fuhrt, einer der nicht sehr zahlreichen deutschen Kenner japanischer Zeitgeschichte, konstatiert hat, noch immer aus ${ }^{21}$.

Die zeitliche Eingrenzung auf die Jahre zwischen 1945 und 1968 ist dabei der deutschen Perspektive geschuldet: Einem in der alten Bundesrepublik sozialisierten Forscher wird es bei dem Thema nicht zuletzt darum gehen, aus der japanischen Entwicklung Erkenntnisse vor allem für eine präzisere Beurteilung der „Vergangenheitsbewältigung“ in der Ära Adenauer und bis in die Zeit der 68er-Bewegung hinein zu gewinnen. Denn zu deren zentralen Motiven wird gängigerweise die Kritik an der „Unfähigkeit zu trauern“ (A. Mitscherlich), also das Ungenügen am gesellschaftlichen Umgang mit der NS-Erblast in den 1950er und frühen 1960er Jahren gezählt ${ }^{22}$, auch wenn sich heute dem „abgeklärten Blick“ manches Altachtundsechzigers selbst die Ära Adenauer retrospektiv ganz anders darstellt, weil sie „bei näherem Hinsehen“ gerade auch bezüglich der „Vergangenheitsbewältigung“ „bemerkenswert konfliktbetont“ gewesen sei und der „Konsens des diskreten Schweigens ... sich ... immer wieder als brüchig“ erwiesen habe ${ }^{23}$. Nachdem die von Ralph Giordano später polemisch zugespitzte 68er-Formel von einer „zweiten Schuld“ der Deutschen wegen ihrer Verdrängungsleistungen nach 1945 den notwendigen Widerspruch erfahren hatte ${ }^{24}$, be-

20 Zur Problematik einer Anwendung des Faschismus-Begriffs auf Japan vgl. Martin, Japan und der Krieg, S. $209 \mathrm{ff}$.

21 Fuhrt, Von der Bundesrepublik lernen?, S. 337.

22 Vgl. etwa Pfeil, Kampf um Geschichtsbilder, S. 248.

23 Die 1950er Jahre verlören somit "alles Idyllische und Beschauliche, alles Verlogene und Restaurative"; so betont unter Verweis auf Wolfgang Kraushaar: Reichel, Vergangenheitsbewältigung, S. 139. Auch Gerd Koenen spricht heute im Rückblick auf die „68er"von ihrem "Knick in der historischen Optik“, also davon, daß sie sich mit dem Restaurationsvorwurf an die "Adenauer-Republik“ in der Zeit und der Gesellschaft, in der sie lebten, „weitgehend vertan und versehen“ haben. Koenen, Das rote Jahrzehnt, S. 101.

24 Siche Hoffmann, Stunden Null?, sowie die Untersuchung der öffentlichen Auseinandersetzung mit dem Nationalsozialismus in der Ära Adenauer durch Kittel, Die Legende, die erstmals breiter dokumentierte, was etwa der einleitende Band von Wolfgang Kraushaars Protestchronik (Kraushaar, Die Protestchronik 1949-1959) später bestätigte: daß sich die 1950er Jahre „als ein Jahrzehnt der politischen Skandale und Kontroversen darstellen, wenn nicht des permanenten politischen Protestes." (Reichel, Vergangenheitsbewältigung, S. 139). Daß zahlreiche einzelne Facetten des öffentlichen Umgangs mit der NS-Vergangenheit weiterer 
gann auch die historische Forschung zu diesem Fragenkomplex, von Thesen und Antithesen zum Versuch von Synthesen überzugehen. So findet sich heute in der wissenschaftlichen Literatur mehr und mehr die Einschätzung, von „einer allgemeinen Verdrängung der Zeit des Nationalsozialismus ..., was die ersten beiden Jahrzehnte der Bundesrepublik angeht“, könne „nicht die Rede sein" 25.

Worauf aber kann zurückgreifen, wer im vergleichenden Blick auf den deutschen Forschungsstand das zum japanischen Fall greifbare Wissen über die Bestrafung der Hauptverantwortlichen nach 1945, die Disqualifizierung belasteter Personen, die Entschädigung der Opfer sowie die politisch-kulturelle Auseinandersetzung mit dem überwundenen Regime und seinen Verbrechen $^{26}$ zu einer ersten knappen Überblicksdarstellung zu bündeln sucht? Lange lagen zum Gesamtkomplex lediglich eine Reihe älterer kleiner Essays vor $^{27}$, so daß das 1994 erschienene Buch des niederländischen Journalisten Ian Buruma ${ }^{28}$ im Grunde genommen die wichtigste Darstellung zum Thema ist. Zwar „weit von einer systematischen Studie entfernt", vermittelt es dennoch in feuilletonistischer Weise wichtige Einsichten „in die psychologischen Hintergründe von Bewältigungs- und Verdrängungsformen in Japan und Deutschland“29. Mehr oder weniger von Buruma inspiriert oder durch die in den 1990er Jahren einsetzende Diskussion um Entschädigung für Zwangsprostituierte der japanischen Armee oder ehemalige Zwangsarbeiter angeregt, sind in den letzten Jahren auch vergleichende Monographien zur amerikanischen Besatzungs- und Schulreformpolitik in Deutschland und Japan bzw. zur Geschichtsschreibung in Westdeutschland und Ja-

Erforschung bedürfen, hat Axel Schildt zu Recht betont. Schildt, Der Umgang mit der NSVergangenheit, S. $23 \mathrm{ff}$.

25 Winkler, Der lange Weg nach Westen. Zweiter Band, S. 176. Eine eigentümliche Ambivalenz des frühen deutschen Umgangs mit dem Nationalsozialismus, „nicht eine allgemeine Verdrängung " konstatiert auch Thamer, NS-Vergangenheit im politischen Diskurs, S. 44. Berghoff, Zwischen Verdrängung und Aufarbeitung, S. 111 u. 114, sieht den Umgang der bundesdeutschen Gesellschaft mit dem „Dritten Reich“ weder von der Verdrängungs- noch von der Aufarbeitungsthese adäquat beschrieben, meint aber, um 1955 herum zumindest das Ende einer „hermetischen Erinnerungsverweigerung“ erkennen zu können. Neben dem sehr breiten Spektrum unterschiedlichster Ambivalenz-Befunde halten sich aber auch Positionen, die den Eindruck erwecken, sich von einer eher meinungsorientierten Betrachtungsweise nicht lösen zu können, obwohl sich die deutschlandpolitischen Pulverdämpfe, die lange über der Frage der „Vergangenheitsbewältigung“ lagen, seit langem verzogen haben. Als Beispiel für die letztere Denkschule genannt, der im Eifer des Gefechts schon einmal gravierende sachliche Fehler unterlaufen (vgl. Jesse, Lesebuch zur Demokratiegründung, S. 530), sei Norbert Frei, Hitlers Eliten nach 1945, S. 312. Dort wird der Schluß nahegelegt, erst mit dem „Machtwechsel“ zur Sozialdemokratie nach der Studentenrevolte seien die Bedingungen für eine wirkliche Bewältigung der NS-Vergangenheit erfüllt gewesen.

26 Vgl. hierzu den von Helmut König unterbreiteten Vorschlag zur Konzeption von Vergangenheitsbewältigung in: König, Von der Diktatur zur Demokratie, S. $378 \mathrm{f}$.

27 Martin, Restauration - Die Bewältigung der Vergangenheit in Japan; Nakai, Die „Entmilitarisierung“ Japans und die „Entnazifizierung“ Deutschlands nach 1945 im Vergleich.

28 Buruma, Erbschaft der Schuld.

${ }^{29}$ Fuhrt, Von der Bundesrepublik lernen?, S. 337. 
pan 1945-196030 erschienen, und auch einige Tagungsbände und Themenhefte haben sich - im Anschluß an einen früheren Sammelband von Arnulf Baring und Masamori Sase ${ }^{31}$ - unterschiedlich intensiv mit einzelnen Facetten des Bewältigungsprozesses in beiden Ländern (mit Fragen nach Kontinuität und Neubeginn 1945, dem Einfluß der Besatzungsmächte auf die deutsche und japanische Rechtsordnung sowie dem Umgang mit Kriegsund Besatzungsunrecht) ${ }^{32}$ beschäftigt. Hervorzuheben sind besonders der von Sebastian Conrad 2001 herausgegebene Themenband des Jahrbuchs für Außereuropäische Geschichte (Periplus) ${ }^{33}$ sowie der gerade (2003) von Christoph Cornelißen, Lutz Klinkhammer und Wolfgang Schwentker veröffentlichte Vergleich der „Erinnerungskulturen“ in Deutschland, Italien und Japan seit $1945^{34}$, wo weitere wichtige Problemfelder von der Rolle des Tennô bis zu den Wirkungen des Staatsshintô angerissen werden, ohne daß die meisten der Beiträge allerdings selbst einen vergleichenden Ansatz verfolgten.

Nicht nur an komparatistischen Synthesen mangelt es bislang. Beim Blick speziell auf das deutschsprachige Schrifttum zum Thema japanische „Vergangenheitsbewältigung“ fiel bis in die jüngste Zeit hinein generell ein „relatives Desinteresse an Japans Umgang mit der eigenen Vergangenheit auf " 35 , und zwar über das durch die Sprachbarriere bedingte Maß hinaus. Japan wurde in der Bundesrepublik - zugespitzt formuliert - meist vor allem als ökonomische Herausforderung oder aber rätselhaft verklärt als Land des Lächelns wahrgenommen, während die historisch-politische Entwicklung oft außer Betracht blieb. Kaum rezipiert worden ist beispielsweise die fundierte Sozialgeschichte der politischen Elite Japans 1945 bis 1965 aus der Feder des langjährigen Sekretärs der deutschen Botschaft in Tokio, die bereits 1973 in den Schriften des Hamburger Instituts für Asienkunde erschienen ist ${ }^{36}$. Auch eine Dokumentation über die amerikanische Besatzungsherrschaft in Japan in den Vierteljahrsheften für Zeitgeschichte $1978^{37}$, die erste Publikation zum Thema in einer deutschen Fachzeitschrift, hat keinen Forschungsschub ausgelöst.

Ulrich Menzel, Herausgeber einer vierbändigen Japan-Anthologie im Suhrkamp-Verlag, klagte denn auch (1989), daß es noch nicht einmal eine

30 Rosenzweig, Erziehung zur Demokratie; Hentschke, Demokratisierung als Ziel; Conrad, Auf der Suche nach der verlorenen Nation.

31 Baring/Masamori (Hg.), Zwei zaghafte Riesen?, mit Einzelbeiträgen auch zu den Themen Reeducation und Entmilitarisierung.

32 Petzina/Ruprecht, Wendepunkt 1945?; Diestelkamp u.a., Zwischen Kontinuität und Fremdbestimmung; Marxen/Miyazawa/Werle, Der Umgang mit Kriegs- und Besatzungsunrecht.

33 Vgl. hierzu die instruktive Einleitung von Conrad, Erinnerungspolitik in Japan.

34 Cornelißen/Klinkhammer/Schwentker, Erinnerungskulturen.

35 Fuhrt, Erzwungene Reue, S. 10.

36 Zahl, Die politische Elite Japans.

37 Benz, Amerikanische Besatzungsherrschaft in Japan. 
von einem deutschen Autor verfaßte, umfassende und grundlegende monographische Darstellung der japanischen Zeitgeschichte gebe, nachdem die traditionell „unpolitisch“-philologisch orientierten deutschen Japanologen es auch nach 1945 unterlassen hätten, eine „Diversifizierung des Faches in Richtung Japankunde anzuregen " 38 . Eine Ausnahmeerscheinung stellt insofern der 1965 eingerichtete Lehrstuhl für Geschichte Japans an der RuhrUniversität Bochum dar, der sich noch heute auf seiner Homepage rühmt, „die einzige Professur im deutschsprachigen Raum“ zu sein, die „so explizit auf die japanische Geschichte ausgerichtet ist“ 39 , ohne allerdings für das hier zu traktierende zeitgeschichtliche Themenfeld besonders viel Einschlägiges hervorgebracht zu haben. Erwähnung verdient deshalb auch das Japan-Seminar der Universität Halle-Wittenberg, wo am Lehrstuhl von Gesine Foljanty-Jost die jüngst veröffentlichte, sehr verdienstvolle, wenn auch aufgrund der Themenstellung für die frühe Phase noch manche Fragen offen lassende Dissertation von Volker Fuhrt über „Vergangenheitsbewältigung“ und Kriegsschulddiskussion in Japan entstanden ist ${ }^{40}$.

Über die lückenhafte deutsche Forschungsliteratur hinaus können der japanischen Sprache Unkundige sich aber vor allem einer ganzen Reihe englischsprachiger, vor allem amerikanischer Studien bedienen, um Informationen über die „Vergangenheitsbewältigung “ im Land der aufgehenden Sonne zu gewinnen, da die - stärker auch geschichts- und sozialwissenschaftlich orientierte - Japanologie in den USA aus leicht nachvollziehbaren geographischen wie historischen Gründen spätestens seit den frühen 1940er Jahren ausgesprochen produktiv ist ${ }^{41}$. Hervorgehoben sei nur - wegen ihres komparatistischen Ansatzes - die frühe Studie von John D. Montgomery über die von der amerikanischen Besatzungsmacht nach 1945 inaugurierten „künstlichen Revolutionen“ in Deutschland und Japan"2.

Auf dieser Basis läßt sich der Versuch unternehmen, von der „Vergangenheitsbewältigung“ eines Landes zu handeln, das aus der Perspektive der

38 Menzel, Im Schatten des Siegers: Japan. Bd. 1, S. 13. Die beklagte Lücke ist allerdings durch Rudolf Hartmanns Geschichte des modernen Japan, die von der Mitte des 19. Jahrhunderts bis in die 1980er Jahre reicht, weitgehend geschlossen worden. R. Hartmann, Geschichte des modernen Japan.

39 http://www.ruhr-uni-bochum.de/gj/index.html (23.03.04).

40 Fuhrt, Erzwungene Reue. Der ebenfalls zu Themen der japanischen Zeitgeschichte vor allem für die Zeit bis zum Zweiten Weltkrieg publizierende Freiburger Historiker Bernd Martin hat eine Professur für Neuere und Neueste Geschichte inne. Vgl. etwa Martin, Japan and Germany, sowie ders., Japans Weg in die Moderne. Sebastian Conrad würdigt Martin in einer Rezension des genannten englischsprachigen Titels als den einzigen Inhaber eines historischen Lehrstuhls an einer deutschen Universität, der sich mit der jüngsten Geschichte Japans befasse und damit eine „rühmliche Ausnahme vom Germano- und Eurozentrismus der Profession" darstelle. Japanstudien 9 (1997), S. 377-384, Zitat S. 377.

41 Wie sehr die amerikanische Literatur dominiert, zeigt auch der HZ-Forschungsbericht von Martin, Japan und der Krieg in Ostasien, der ein eigenes Kapitel über Kriegsverbrecherprozesse und Besatzungspolitik enthält (S. 189-209).

42 Montgomery, Forced to be Free. 
deutschen Zeitgeschichte auch deshalb besonders interessant ist, weil das fernöstliche Inselreich, ein Land mit einzigartiger ästhetischer Tradition und unvergleichlicher Feinheit im menschlichen Umgang wie im Sprachgefühl, in den 1930er und 1940er Jahren durch die Beseitigung demokratischer Liberalität im Inneren und kriegerische Politik nach außen „einer ähnlichen Politik nationalistischer Verirrungen gefolgt war wie Deutschland“ 43 , das doch eigentlich als Land der Dichter und Denker galt. Die von Bernd Martin herausgearbeiteten „faszinierenden Parallelen" 44 zwischen deutschem und japanischem „Sonderweg" bis 1945 sollten sich nach dem Zweiten Weltkrieg jedoch beträchtlich verschieben.

In methodischer Hinsicht versteht sich die vorliegende Arbeit - nach der von Jürgen Osterhammel in seinen Bemerkungen zur transkulturell vergleichenden Geschichtswissenschaft vorgeschlagenen Typologie - als Partialvergleich ${ }^{45}$. Nicht die gesamte Geschichte der japanischen und (bundes-) deutschen Gesellschaft in den Dekaden nach dem Zweiten Weltkrieg wird einer komparativen Prozedur unterzogen, vielmehr richtet sich die Erkenntnisabsicht auf das - immer noch breite, aber doch besser überschaubare-Segment der „Vergangenheitsbewältigung“. Gerade Partialvergleiche zwischen nicht mehr als zwei Fällen - erfolgreich durchgeführt etwa für den nahezu zeitgleichen ländlichen antikapitalistischen Protest in Mexiko und der Ukraine ${ }^{46}$ - lassen sich am ehesten mit dem „Handwerksethos des Fachhistorikers" vereinbaren. Für den Historiker der deutschen „Vergangenheitsbewältigung“ gilt dabei, was Jürgen Osterhammel über den Fachmann für die britische Industrialisierung bemerkt hat: Dieser verspreche sich tiefere Einsichten in sein eigenes Spezialgebiet von einem Vergleich mit Japan und werde - ohne über dieses Land aus den Quellen gearbeitet zu haben doch wissen, welche Fragen an die Sekundärliteratur zu stellen seien. Und er werde zudem keine unüberwindlichen Kulturbarrieren überwinden müssen, um „Zugang zu der umfangreichen englischsprachigen Forschung über die industrielle Entwicklung Japans [zu] finden - auch wenn er sich mit einer gewissen Demut seiner fehlenden Sprachkenntnisse bewußt sein muß." 47 Die Demut erstreckt sich - angesichts der oft hochgespannten Erwartungen an den transkulturellen Vergleich - „auch auf den embarras de

43 Broszat, Zeitgeschichte in Japan, S. 289.

44 So der Klappentext zu Martins Studie "Japan and Germany“.

45 Osterhammel, Transkulturell vergleichende Geschichtswissenschaft, S. 294.

46 Dahlmann, Land und Freiheit. Vgl. auch den deutsch-französischen Vergleich von Kittel, Provinz zwischen Reich und Republik.

47 Osterhammel, Transkulturell vergleichende Geschichtswissenschaft, S. 295. Die japanische Sprachbarriere ist übrigens auch von dem erwähnten Forschungsbericht Bernd Martins im Traditionsorgan der deutschen Geschichtswissenschaft nicht überwunden worden, der sich ebenfalls dadurch legitimicrt, daß zu dem Thema von deutschen Japanologen jahrzehntelang nicht hinreichend viel vorgelegt wurde. 
richesse der weltweiten Geschichtsforschung “48, deren sämtlichen spezialistischen Verästelungen im Rahmen eines komparatistischen Essays allerdings weder nachgestiegen werden kann noch muß ${ }^{49}$.

Der Begriff „Vergangenheitsbewältigung“ soll in dieser Untersuchung, die sich vor allem an einen deutschen Leserkreis richtet, trotz seiner bekannten Problematik Verwendung finden. Denn zum einen wissen bei einem Terminus selten „so viele, was gemeint ist“, auch ohne genau „zu wissen, was er meint" ${ }^{\text {50; }}$ zum anderen ließe sich mit Helmut König argumentieren, der den Begriff für umfassender hält als dessen Alternativen wie „Aufarbeitung der Vergangenheit", „Erinnerungskultur“ oder „Vergangenheitspolitik“, weil er das „gesamte Spektrum der politischen, kulturellen, juristischen, wissenschaftlichen, pädagogischen, ästhetischen und religiösen Dimensionen des Themas" bezeichne ${ }^{51}$. Allerdings könnte man die genannten Felder ebenso gut unter den - freilich weit verstandenen - Begriff der „Erinnerungskultur" fassen ${ }^{52}$, der im Zuge des kulturalistischen Trends in der Geschichtswissenschaft nachgerade hyperinflationäre Verbreitung gefunden hat, auch wenn "Erinnerungskultur" im engeren Sinne eigentlich nur „die wissenschaftlich-dokumentarische, die ästhetisch-expressive und die feierlich-rituelle Auseinandersetzung mit der Vergangenheit " 53 meint. Dennoch besitzt der Begriff gegenüber dem der „Vergangenheitsbewältigung“ schon den praktischen sprachlichen Vorzug, auch besser adjektivisch verwendet werden zu können. Im folgenden sollen beide parallel in dem von König beschriebenen Sinne benutzt werden, während auf den Terminus „Vergangenheitspolitik“ (N. Frei) verzichtet wird. Im Kontext einer Arbeit über die politisch-legislativen Voraussetzungen der Ahndung von NS-Straftaten in den Jahren 1949 bis 1954 geprägt, die unser Wissen um Entscheidungsprozesse, Einflußstrukturen und Diskussionszusammenhänge in einigen Feldern der Erinnerungskultur zweifelsohne vermehrt hat ${ }^{54}$, suggeriert der Begriff „Vergangenheitspolitik“ die strategische Planung eines geschlossenen politischen Komplexes durch Regierungshandeln in einem Maße, das

48 Osterhammel, Transkulturell vergleichende Geschichtswissenschaft, S. 283.

49 Dies gilt aufgrund des gewählten Ansatzes vor allem für die kaum mehr überschaubare Literatur zur deutschen „Vergangenheitsbewältigung“, die hier nur punktuell für die ausgewählten Vergleichsebenen herangezogen wird, also etwa nicht für die nur am Rande behandelte, weil in Japan kaum eine Rolle spielende Rezeption des Widerstandes nach 1945. Wer einen Überblick über die Auseinandersetzung mit der NS-Diktatur in der Bundesrepublik gewinnen möchte, kann im übrigen bereits auf die ausgewogene Darstellung von Reichel, Vergangenheitsbewältigung, zurückgreifen.

50 Brochhagen, Nach Nürnberg, S. 11.

51 König, Die Zukunft der Vergangenheit, S. 8.

52 Vgl. J. Assmann, Das kulturelle Gedächtnis; Nora, Zwischen Geschichte und Gedächtnis; A. Assmann, Erinnerungsräume; Hockerts, Zugänge zur Zeitgeschichte; Cornelißen, Was heißt Erinnerungskultur?

53 Reichel, Politik mit der Erinnerung, S. $26 \mathrm{f}$.

54 Frei, Vergangenheitspolitik, S. 12. 
es auf den weiten und historisch verminten Feldern der „Vergangenheitsbewältigung" in einer offenen Gesellschaft so kaum geben konnte. Auch wenn der Vater des Begriffs selbst betont hat, hinter den von ihm untersuchten Parlamentsinitiativen, Gesetzgebungswerken und administrativen Entscheidungen zur „Bewältigung der frühen NS-Bewältigung“ sei keine strategische Planung $\mathrm{zu}$ erkennen ${ }^{55}$, hat er zu diesem Mißverständnis durch seine Schwerpunktsetzung und die Art mancher Urteile doch Anlaß gegeben. Mit „Vergangenheitspolitik“, so bemerkte folglich etwa Andreas Mink zu Freis Arbeit zustimmend, „bezeichnen wir seither die Bemühungen in der frühen Adenauer-Ära, durch weitreichende Amnestie-Gesetze aus Hitlers ,Volksgemeinschaft' eine Gesellschaft der Ahnungslosen und der Mitläufer zu formen." 56

Eher noch als von „Vergangenheitspolitik“ könnte man für die Ära Adenauer vielleicht von „Bewältigungspolitik“ sprechen, um zumindest näher an die semantische Problematik des zeitgenössischen, Mitte der 1950er Jahre aufkommenden Begriffes „Vergangenheitsbewältigung“ anzuknüpfen, auch wenn das manchem zu historistisch vorkommen mag. Charakteristisch für die (bundes-)deutsche Entwicklung scheint der Terminus „Vergangenheitsbewältigung“ jedoch insofern, als er ein Ziel vorgab, das gerade in bezug auf die NS-Zeit von vornherein nicht zu erreichen war - denn, was geschehen ist, läßt sich nicht mehr „bewältigen“ -, so daß jedes politische Handeln auf diesem Feld hinter dem begrifflichen Anspruch zurückbleiben mußte und spätere Diagnosen des Scheiterns geradezu vorprogrammiert waren ${ }^{57}$.

Dagegen hat Sebastian Conrad im vergleichenden Blick auf Japan aus ganz anderen Gründen zur Vorsicht bei der Verwendung des deutschen Terminus „Vergangenheitsbewältigung“ gemahnt: Der Begriff rede die Möglichkeit eines „richtigen“ Umgangs mit einer „schlimmen“ Vergangenheit ein und strukturiere den Blick auf die japanischen Diskussionen in einer Weise vor, „die in der Regel lediglich dazu führt, Defizite zu konstatieren" 58 . Conrads Monitum ist insofern nachvollziehbar, als Mitte der 1980er Jahre im Zeichen vermehrter Kritik an den Defiziten nationaler Erinnerungskultur in Japan immer wieder - oft mit Bezug auf Richard von Weiz-

55 Ebd., S. 13.

56 Andreas Mink, Zweierlci Kontinuität, in: Aufbau, New York-Berlin, issue 25, December 12, 2002 (http://www.aufbauonline.com/2002/issue25/1_2.html).

57 Kittel, Die Legende, S. 21-28. Nach Reichel, Vergangenheitsbewältigung, S. 20, offenbart sich in dem vieldeutigen, gewichtigen, als typisch deutsch geltenden Wort vielleicht auch eine trotzig-naive „Sehnsucht nach Unmöglichem“ (Bernhard Schlink), die das Vergangene so in Ordnung bringen wollc, daß seine Erinnerung nicht mehr auf der Gegenwart laste. Zur Problematik des umstrittenen Begriffs vgl. auch Dudek, „Vergangenheitsbewältigung“.

58 Conrad, Erinnerungspolitik in Japan, S. 4. Conrad schlägt statt dessen vor, von „Erinnerungspolitik“ zu reden, wobei er im Blick etwa auf die diskursive Koalition zwischen USBesatzungsmacht und japanischen Linksintellektuellen Wert darauf legt, „Erinnerungspolitik“ immer auch als „Teil einer gemeinsamen, transnationalen Geschichte" zu verstehen. 
säckers Rede zum 8. Mai 1985 und manchmal sehr pauschal - die Bundesrepublik als das große Vorbild gepriesen wurde. Dennoch läßt sich in dem oben umrissenen offenen Sinn mit dem Begriff „Vergangenheitsbewältigung" arbeiten, zumal der entsprechende japanische Terminus kako no kokufuku, den 1992 eine führende Tageszeitung popularisierte, den Versuch einer direkten Übersetzung aus dem Deutschen darstellt ${ }^{59}$.

Obendrein geht es im folgenden nicht um das - von Conrad zu Recht als unzureichend abgelehnte - bloße Konstatieren von Defiziten der japanischen Erinnerungskultur vor dem Hintergrund einer idealisierten bundesdeutschen Vergleichsfolie; auch wenn der vergleichende Historiker natürlich kaum ganz davon absehen kann, die Unterschiede in den Ergebnisbefunden selbst ebenfalls zu benennen. Darüber hinaus steht aber vor allem die Erklärung der vorgefundenen Stärken und Schwächen des Umgangs mit einer dunklen Vergangenheit in beiden Ländern im Mittelpunkt. Lassen sich die tiefgreifenden Unterschiede tatsächlich auf mentalitäre oder zumindest auf im weitesten Sinne politisch-kulturelle Ursachen zurückführen, wie man es im Anschluß an die verkürzende Formel von Ruth Benedict über die Divergenzen zwischen shintôistischer Scham- und christlicher Schuldkultur tun könnte?60 Welche Rolle spielte dabei die Tatsache, daß es neben Nanking auch noch Hiroshima gab, das immer wieder als "atomarer Holocaust“ charakterisiert wurde ${ }^{61}$ und Japan zu einer weltweit Empathie auslösenden Opfernation schlechthin werden ließ, während nach Auschwitz der Untergang Dresdens oder der Wilhelm Gustloff, Symbole des „konventionellen“ Bombenkriegs und der großen Vertreibung, bei allem unaussprechlichen Schrecken erinnerungskulturell doch keine annähernd vergleichbare Wirkung entfalteten? Darüber hinaus ist zu fragen, ob der von Volker Fuhrt für Japan betonte Faktor des - von den 1950er bis in die 1970er Jahre hinein weithin mangelnden - Außendrucks (gaiatsu) ${ }^{62}$, auch für die abweichende Entwicklung der bundesdeutschen „Vergangenheitsbewältigung“ aufschließende Kraft hat; in dem Sinne, daß foreign pressure - einschließlich nicht zielgerichteter Einflußnahme etwa durch die öffentliche Meinung des Auslands bzw. deren Wahrnehmung in der Bundesrepublik ${ }^{63}$ - hier bereits in den ersten Dekaden nach 1945 entscheidend höher war? Und schließlich: Wie war das Verhältnis zwischen diesem Außendruck und den jeweiligen „inneren Rezeptoren“ der Erinnerungskultur in Japan und Westdeutschland?

59 Ebd., S. 8.

60 Benedict, The Chrysanthemum, S. 223.

61 Zur Problematik dieses Begriffs vgl. Kapitel IV.

62 Fuhrt, Erzwungene Reue. S. $193 \mathrm{f}$;; auch Sebastian Conrad hat dafür plädiert, den japanischen Umgang mit der Vergangenheit „in einem Kontext transnationaler Einflußnahme und Erwartungshaltungen" zu situieren. Conrad, Krisen der Moderne?, S. 177.

$63 \mathrm{Vgl}$. zu diesem erweiterten Konzept von foreign pressure: Miyaoka, Foreign Pressure, S. 10. 